\title{
15 Beschäftigtendatenschutz bei der Sekundärnutzung von Behandlungsdaten
}

\author{
Unter welchen rechtlichen Bedingungen ist der Beschäftigtendatenschutz bei \\ der Sekundärnutzung von Behandlungsdaten zu beachten? Auf welcher Ebene \\ müsste in die Verarbeitung personenbezogener Mitarbeiterdaten ggf. eingewil- \\ ligt werden (z.B. Einrichtungsleitung, Betriebsrat, Betroffene)?
}

Hintergrund dieser Frage ist, dass von den Behandlungsdaten nicht nur Patienten, sondern auch Beschäftigte betroffen sein können. Dies ist dann der Fall, wenn das behandelnde Personal in den für Zwecke der Qualitätssicherung oder Forschung herangezogenen Datensätzen genannt ist. Eine Nennung des behandelnden Personals in der (elektronischen) Patientenakte ist dabei sogar typisch, um Ansprechpartner und Verantwortlichkeiten klarzustellen. Eine Herausnahme dieser Personaldaten ${ }^{918}$ bei der Zweckänderung hin zu Qualitätssicherung und Forschung wird bei zunächst unstrukturierten Daten (wie eingescannten Arztbriefen) schwierig und im Übrigen unter Umständen - gerade bei der Qualitätssicherung - nicht gewollt sein, um dem verantwortlichen Behandlungspersonal eine Rückmeldung geben zu können. Dann ist aber mangels Anonymisierung des Personalbezugs der Beschäftigtendatenschutz auch bei der Sekundärnutzung der Behandlungsdaten zu beachten.

918 Zu diesen Personaldaten gehören identifizierende (Stamm-)Daten wie der Name sowie die Tatsache der Mitwirkung an der Behandlung und ggf. weitere Details zu konkret verantworteten Maßnahmen. 
Dabei wird jedoch zu berücksichtigen sein, dass der Beschäftigtendatenschutz zwar im geltenden Recht einen höheren Stellenwert als der allgemeine Datenschutz haben mag, allerdings keinen so hohen wie der Patientendatenschutz, der zudem durch die Schweigepflicht der Heilberufe flankiert wird. Diese Schweigepflicht ist jedoch für die vorliegende Fragestellung irrelevant. Allerdings ist eine datenschutzrechtliche Erlaubnis für die beschriebene Verwendung der Beschäftigtendaten erforderlich. ${ }^{919}$

Auf die Sonderproblematik, wenn ein Beschäftigter einer Behandlungseinrichtung gleichzeitig deren Patient ist, wird im Folgenden nicht eingegangen. Hier sind in jedem Fall auch die Maßgaben des Patientendatenschutzes und der Schweigepflicht zu beachten, gegebenenfalls aber angesichts der doppelten Abhängigkeit - als Patient und Beschäftigter - zusätzliche Schutzvorkehrungen zu treffen. ${ }^{920}$

Auf (private) Arztpraxen finden auch im Hinblick auf den Beschäftigtendatenschutz einheitlich die Vorschriften des BDSG für nicht-öffentliche Stellen Anwendung, vorrangig also $\$ 32$ BDSG.

In der nachfolgenden Übersicht wird zudem das auf Beschäftigungsverhältnisse in Kliniken vorrangig anwendbare Datenschutzrecht dargestellt. Dabei werden zunächst die vorrangig gültigen Paragrafen des jeweils anwendbaren Gesetzes genannt. Soweit diese Paragrafen, wie beispielsweise $\$ 32$ BDSG, keine abschließende Regelung treffen, kann jedoch auch auf andere Erlaubnistatbestände des entsprechenden Gesetzes oder anderer anwendbarer Gesetze ${ }^{921}$ zurückgegriffen werden.

Auch kommen die Regelungen des kollektiven Arbeitsrechts mit normativer Wirkung, also insbesondere Betriebs- bzw. Dienstvereinbarungen nach dem Betriebsverfassungsgesetz (BetrVG) bzw. den Personalvertretungsgesetzen des Bundes und der Länder oder der Mitarbeitervertretungsgesetze der Kirchen, als datenschutzrechtliche Erlaubnisse in Betracht. ${ }^{222}$ Zusammenfassend lässt sich festhalten, dass solche Betriebs- bzw. Dienstvereinbarungen die Rechtssicherheit von Maßnahmen der Qualitätssicherung und Forschungsvorhaben, soweit von diesen (personenbezogene) Beschäftigtendaten betroffen sind, merklich erhöhen und jedenfalls für die externe Qualitätssicherung sowie die Datenübermittlung zu Forschungszwecken zu empfehlen sind.

Überwiegend finden die Vorschriften des BDSG für nicht-öffentliche Stellen Anwendung, selbst in Kliniken, auf welche das jeweilige Bundesland bezüglich des Patientendatenschutzes sein LKHG erstreckt, da im Übrigen meist auf die jeweils geltenden Vorschriften verwiesen bzw. explizit die Geltung der Regelungen des BDSG für nichtöffentliche Stellen angeordnet wird. Teils erstrecken Bundesländer aber im Hinblick auf den Beschäftigtendatenschutz ihr LDSG sogar auf Kliniken privater Träger. ${ }^{923}$

919 Obgleich in Bezug auf die Beschäftigten, je nach Ausgestaltung des Beschäftigungsverhältnisses und des Datenumgangs, nicht zwingend eine Zweckänderung vorliegt.

920 Besondere Schutzvorkehrungen nicht nur, aber gerade auch für einen solchen Fall (HIV-erkrankte Mitarbeiterin in einem Krankenhaus) fordert EGMR, Urt. v. 17.07.2008 - 20511/03 (I../. Finnland), aufgrund des durch Art. 8 EMRK geschützten Privatlebens.

921 Teils wird ergänzend explizit auf die Landesbeamtengesetze verwiesen, so in $\$ 20$ Abs. 1 LDSG HB.

922 Dies gilt auch für Arztpraxen soweit sie in den Anwendungsbereich des BetrVG fallen und einen Betriebsrat haben, was nicht ausgeschlossen, in der Praxis aber eher selten ist und wenn, dann eher bei größeren MVZ vorkommen dürfte.

923 Was man aus Kompetenzgründen als verfassungsrechtlich bedenklich ansehen kann, vorliegend aber akzeptiert werden soll. 


\section{1 Übersicht 7: Auf Beschäftigungsverhältnisse in Kliniken vorrangig anwendbares Datenschutzrecht (s. Tab. 9)}

Tab. 9 Übersicht 7: Auf Beschäftigungsverhältnisse in Kliniken vorrangig anwendbares Datenschutzrecht

\begin{tabular}{|c|c|c|c|c|c|c|}
\hline \multirow{2}{*}{$\begin{array}{l}\text { Trägerschaft } \\
\text { und Art der } \\
\text { Klinik }\end{array}$} & \multicolumn{2}{|c|}{ Öffentliche Träger } & \multicolumn{2}{|c|}{ Private Träger } & \multicolumn{2}{|c|}{ Kirchliche Träger } \\
\hline & $\begin{array}{l}\text { Bund oder diesem } \\
\text { zugeordnete } \\
\text { Anstalten, } \\
\text { Körperschaften, } \\
\text { Stiftungen }\end{array}$ & $\begin{array}{l}\text { Land oder diesem } \\
\text { zugeordnete } \\
\text { Anstalten, } \\
\text { Körperschaften, } \\
\text { Stiftungen }\end{array}$ & $\begin{array}{l}\text { Plankrankenhaus } \\
\text { (in den jeweiligen } \\
\text { Krankenhausplan } \\
\text { aufgenommen) }\end{array}$ & $\begin{array}{l}\text { Reine Privatklinik } \\
\text { (\$ } 5 \text { Nr. } 2 \text { KHG: } \\
40 \% \text {-Grenze nach } \\
\$ 67 \text { A0 nicht } \\
\text { erfüllt) }\end{array}$ & Evangelisch & Katholisch \\
\hline \multirow{2}{*}{$\begin{array}{l}\text { Baden- } \\
\text { Württemberg }\end{array}$} & \multirow[t]{2}{*}{$\$ 32 \mathrm{BDSG}$} & $\begin{array}{l}\text { Mit eigener } \\
\text { Rechtspersönlichkeit: } \\
\S 32 \text { BDSG }\end{array}$ & \multirow[t]{2}{*}{ \32 BDSG } & \multirow[t]{2}{*}{$\$ 32 \mathrm{BDSG}$} & \multirow[t]{2}{*}{$\S 24$ DSG-EKD } & \multirow[t]{2}{*}{$\S 10 \mathrm{a}$ KDC } \\
\hline & & $\begin{array}{l}\text { Ohne diese: } \$ 36 \\
\text { LDSG }\end{array}$ & & & & \\
\hline Bayern & $\$ 32 \mathrm{BDSG}$ & $\$ 32$ BDSG & $\$ 32 \mathrm{BDSG}$ & $\$ 32$ BDSG & $\S 24$ DSG-EKD & $\$$ 10a KDO \\
\hline Berlin & $\$ 32$ BDSG & $\$ 32$ BDSG & $\$ 32 \mathrm{BDSG}$ & $\$ 32 \mathrm{BDSG}$ & $\$ 24$ DSG-EKD & S 10a KDO \\
\hline Brandenburg & $\$ 32$ BDSG & $\$ 29$ LDSG & $\$ 29$ LDSG & \$2 29 LDSG & $\S 24$ DSG-EKD & \ 10a KDO \\
\hline Bremen & $\$ 32$ BDSG & $\$ 20$ LDSG & $\$ 20$ LDSG & $\S 20$ LDSG & $\S 24$ DSG-EKD & \10a KDO \\
\hline Hamburg & $\$ 32$ BDSG & $\begin{array}{l}\text { Private Rechtsform: } \\
\$ 32 \text { BDSG } \\
\text { Öffentl. Rechtsform: } \\
\$ 28 \text { LDSG }\end{array}$ & $\$ 32 \mathrm{BDSG}$ & $\$ 32$ BDSG & \24 DSG-EKD & \10a KDO \\
\hline Hessen & $\$ 32 \mathrm{BDSG}$ & $\$ 34$ LDSG & $\$ 34$ LDSG & $\$ 34$ LDSG & $\S 24$ DSG-EKD & \10a KDO \\
\hline $\begin{array}{l}\text { Mecklenburg- } \\
\text { Vorpommern }\end{array}$ & $\$ 32 \mathrm{BDSG}$ & $\$ 35$ LDSG & $\$ 35$ LDSG & $\$ 35$ LDSG & $\$ 24$ DSG-EKD & $\$$ 10a KDO \\
\hline $\begin{array}{l}\text { Nieder- } \\
\text { sachsen }\end{array}$ & $\$ 32$ BDSG & $\$ 32$ BDSG & $\$ 32 \mathrm{BDSG}$ & $\$ 32$ BDSG & $\$ 24$ DSG-EKD & \10a KDO \\
\hline $\begin{array}{l}\text { Nordrhein- } \\
\text { Westfalen }\end{array}$ & $\$ 32$ BDSG & $\$ 29$ LDSG & $\$ 29$ LDSG & $\$ 32 \mathrm{BDSG}$ & $\S 24$ DSG-EKD & \$ 10a KDO \\
\hline $\begin{array}{l}\text { Rheinland- } \\
\text { Pfalz }\end{array}$ & $\$ 32$ BDSG & $\$ 31$ LDSG & $\$ 32 \mathrm{BDSG}$ & $\$ 32 \mathrm{BDSG}$ & $\$ 24$ DSG-EKD & S 10a KDO \\
\hline Saarland & $\$ 32 \mathrm{BDSG}$ & $\$ 31$ LDSG & $\$ 32 \mathrm{BDSG}$ & $\$ 32$ BDSG & $\S 24$ DSG-EKD & \10a KDO \\
\hline Sachsen & $\$ 32$ BDSG & $\begin{array}{l}\text { Mit eigener } \\
\text { Rechtspersönlichkeit: } \\
\$ 32 \text { BDSG } \\
\text { Ohne diese: } \$ 37 \\
\text { LDSG }\end{array}$ & $\$ 32 \mathrm{BDSG}$ & $\$ 32 \mathrm{BDSG}$ & $\S 24$ DSG-EKD & \10a KDO \\
\hline $\begin{array}{l}\text { Sachsen- } \\
\text { Anhalt }\end{array}$ & $\$ 32 \mathrm{BDSG}$ & \$28 LDSG & $\$ 32 \mathrm{BDSG}$ & $\S 32 \mathrm{BDSG}$ & $\$ 24$ DSG-EKD & \10a KDO \\
\hline $\begin{array}{l}\text { Schleswig- } \\
\text { Holstein }\end{array}$ & $\$ 32 \mathrm{BDSG}$ & $\begin{array}{l}\text { Private Rechtsform: } \\
\$ 32 \text { BDSG } \\
\text { Öffentl. Rechtsform: } \\
\$ 23 \text { LDSG }\end{array}$ & $\$ 32 \mathrm{BDSG}$ & $\$ 32 \mathrm{BDSG}$ & $\S 24$ DSG-EKD & \10a KDO \\
\hline Thüringen & $\$ 32 \mathrm{BDSG}$ & $\$ 32 \mathrm{BDSG}$ & $\$ 32 \mathrm{BDSG}$ & $\$ 32 \mathrm{BDSG}$ & \ 24 DSG-EKD & \ 10a KDO \\
\hline
\end{tabular}




\subsection{Anwendungsbereich des BDSG}

Zunächst sollen die Vorschriften des BDSG für nicht-öffentliche Stellen, wie sie für Arztpraxen, Kliniken des Bundes sowie Kliniken vieler anderer Träger gelten, in dieser Hinsicht untersucht werden.

\subsubsection{Datenschutzrechtliche Erlaubnis}

\subsubsection{Zweckbestimmung des Beschäftigungsverhältnisses ( $\$ 32$ BDSG)}

Personenbezogene Daten eines Beschäftigten dürfen für Zwecke des Beschäftigungsverhältnisses nach $\mathbb{} 32$ Abs. 1 S. 1 BDSC erhoben, verarbeitet und genutzt werden, wenn dies für die Entscheidung über die Begründung eines Beschäftigungsverhältnisses oder nach Begründung für dessen Durchführung oder Beendigung erforderlich ist.

Es stellt sich also mit anderen Worten die Frage, ob die Verarbeitung von Arbeitnehmerdaten, die zwar nicht zur Durchführung des Arbeitsverhältnisses erforderlich sind, aber gleichwohl zur Erfüllung von sich aus der Arbeitgeberstellung ergebenden berechtigten Interessen benötigt werden, nach der gesetzlichen Neuregelung in $\$ 32$ Abs. 1 S. 1 BDSG untersagt sein soll. ${ }^{924}$ Entgegen seinem Wortlaut ist $\mathbb{3} 32$ Abs. 1 S. 1 BDSG nach den Gesetzesmaterialien auch dann einschlägig, wenn der Arbeitgeber seine im Zusammenhang mit der Durchführung des Arbeitsverhältnisses bestehenden Rechte wahrnimmt, z.B. durch Ausübung seines Weisungsrechts oder durch Kontrolle der Leistung oder des Verhaltens des Beschäftigten. ${ }^{925}$ Der Arbeitgeber kann sich also auch für alle Maßnahmen, die im Zusammenhang mit dem Beschäftigungsverhältnis stehen und die der Erfüllung eigener Pflichten oder der Durchsetzung eigener Rechte dienen, auf die Rechtfertigung zur Datenverarbeitung aus $\mathbb{3} 32 \mathrm{Abs}$. 1 Satz 1 BDSG berufen. ${ }^{926}$

Fraglich ist zunächst, ob dies bei der Qualitätssicherung noch der Fall ist. Dies wird zunächst bei gesetzlich zwingend vorgeschriebenen oder explizit auch im Hinblick auf den Datenumgang (ohne Beschränkung auf Patientendaten, wie er allerdings für die LKHG üblich ist) erlaubten Maßnahmen zur Sicherung der Behandlungsqualität der Fall sein (z.B. nach $\$ 299$ SGB V).

Wenn die Qualitätssicherung beschäftigtenbezogen erfolgen soll, um eine individuelle Leistungskontrolle zu ermöglichen, ${ }^{927}$ wird man einen entsprechenden Bezug zum Beschäftigungsverhältnis bejahen können. Zu beachten wäre dann aber die regelmäßig bestehende Mitbestimmungspflicht des Betriebs- oder Personalrats bzw. der Mitarbeitervertretung. ${ }^{928}$ Andernfalls, also wenn eine beschäftigtenbezogene Leistungskontrolle zwar unter Umständen möglich, aber nicht beabsichtigt ist, greift zwar wohl ebenfalls die Mitbestimmungspflicht, nicht aber der Erlaubnistatbestand nach $\$ 32$ Abs. 1 S. 1 BDSG.

924 Vgl. Gola/Jaspers, RDV 2009, 212.

925 Vgl. Thüsing, NZA 2009, 865, 867; Schmidt, RDV 2009, 193, 197; Albrecht, jurisPR-ITR 20/2009 Anm. 2.

926 Schmidt, RDV 2009, 193, $197 f$.

927 Die Verhaltenskontrolle spielt in vorliegendem Kontext keine Rolle.

928 S. dazu unten Kap. I.15.2.2, S. 329f. 
Die beschäftigtenbezogene Verarbeitung von Behandlungsdaten zu Forschungszwecken wird hingegen im Allgemeinen kaum als Leistungskontrolle im genannten Sinn eingestuft werden können, weshalb insoweit in der Regel $\mathbb{3} 32$ Abs. 1 S. 1 BDSG als Erlaubnistatbestand ausscheidet.

Soweit das Beschäftigungsverhältnis allerdings einen expliziten Bezug zur Forschung aufweist, dürfte von dessen Zweckbestimmung nach $\mathbb{3} 32 \mathrm{Abs}$. 1 S. 1 BDSG auch die Verwendung von beschäftigtenbezogenen Behandlungsdaten zu Forschungszwecken umfasst sein. ${ }^{229}$ Dies dürfte beispielsweise in Universitätskliniken nicht selten sein, zu deren Auftrag neben der Versorgung ausdrücklich auch die wissenschaftliche Forschung gehört. Ob insoweit die vorsorgliche Aufnahme eines Forschungsbezugs in einen Arbeitsvertrag ausreicht, obgleich der Beschäftigte in aller Regel nicht mit Forschung in Berührung kommt, kann jedoch bezweifelt werden.

\subsubsection{Zweckänderungen}

Soweit die Zweckbestimmung des Beschäftigungsverhältnisses den Datenumgang zur Qualitätssicherung oder Forschung nicht umfasst, liegt eine besonders zu rechtfertigende Zweckänderung vor.

\section{Verhältnis von § 32 BDSG zu anderen Erlaubnistatbeständen}

Als Erlaubnisnorm für im Beschäftigungsverhältnis benötigte Daten tritt $\$ 32$ BDSC an die Stelle des allgemeiner gefassten Zulässigkeitstatbestandes des $\mathbb{2} 28$ Abs. 1 S. 1 Nr. 1 BDSG (nach Maßgabe der Zweckbestimmung eines Vertragsverhältnisses), welcher nun durch $\sqrt{32}$ BDSG konkretisiert wird. $930 \$ 32$ BDSG enthält jedoch keine abschließende Regelung des Arbeitnehmerdatenschutzes. ${ }^{931}$ Dies bedeutet, dass die übrigen, nicht verdrängten Erlaubnistatbestände aus $\$ 28$ BDSG auch auf Beschäftigtendaten Anwendung finden können, ${ }^{932}$ was von Bedeutung ist, wenn man nicht bereits eine Rechtfertigung nach $\mathbb{3 2}$ BDSG annimmt.

\section{Qualitätssicherung}

Die Qualitätssicherung dient der Wahrung berechtigter Interessen der verantwortlichen Stelle nach $\mathbb{2} 28$ Abs. 1 S. 1 Nr. 2 BDSG, zu denen auch eine Zweckänderung nach $\mathbb{2} 8$ Abs. 2 Nr. 1 BDSG herbeigeführt werden kann. ${ }^{933}$ Daher dürfen auch Beschäftigtendaten, welche im Rahmen von Beschäftigungs- und Behandlungsverhältnissen angefallen sind, grundsätzlich für Zwecke der Qualitätssicherung verwendet werden, soweit dies erforderlich ist. Von einer Erforderlichkeit wird man ausgehen

929 Vgl. die Argumentation bei Arbeitsverhältnissen, die sich auf einen Gesamtkonzern und nicht nur eine Konzerngesellschaft beziehen, also solche mit klarem Konzernbezug: Büllesbach, in: Roßnagel (Hrsg.), Handbuch Datenschutzrecht, Kap. 6 Rdnr. 67

930 Gola/Schomerus, BDSG, $\$ 32$ Rdnr. 2. Dagegen hält Seifert, in: Simitis (Hg.), BDSG, $§ 32$ Rdnr. 17, den Rückgriff, insbes. auf $\$ 28$ Abs. 1 S. 1 Nr. 2 BDSG, grundsätzlich für ausgeschlossen.

931 Gesetzesbegründung, BT-Drucks. 16/13657, S. 34f.; Gola/Schomerus, BDSG, § 32 Rdnr. 2.

932 So zur Verarbeitung von Beschäftigtendaten, die nicht dem Beschäftigungsverhältnis im engeren Sinne dienen: BAG, Urt. v. 07.02. 2012 - 1 ABR 46/10, RDV 2012, 192: Das Erheben von Daten über die krankheitsbedingten Fehlzeiten durch den Arbeitgeber und ihre Übermittlung an den Betriebsrat ist auch bei fehlender Zustimmung der betroffenen Arbeitnehmer nach $₫ 28 \mathrm{Abs} .6 \mathrm{Nr}$. 3 BDSG zulässig, u.a. für Zwecke des betrieblichen Eingliederungsmanagements (Rdnr. 24). Allerdings ist die genaue Abgrenzung zwischen $₫ 32$ BDSG und der allgemeinen Vorschrift des $\$ 28$ BDSG mit seinen verschiedenen Erlaubnistatbeständen in der juristischen Literatur, vor allem kurz nach Verabschiedung des $§ 32$ BDSG, kontrovers diskutiert worden, vgl. Schmidt, RDV 2009, 193, 195. Allgemein zu dieser Erlaubnisnorm: Gola/Schomerus, BDSG, § 28 Rdnr. $24 f f$. 
können, wenn eine vorgängige Anonymisierung (Herausnahme der Personaldaten) mit unverhältnismäßigem Aufwand verbunden wäre (wovon man bei unstrukturierten Scans ausgehen kann) oder wenn (patienten-)individuelle Rückmeldungen gegeben werden sollen. ${ }^{934}$ Weitere Voraussetzung nach $\$ 28$ Abs. 1 S. 1 Nr. 2 BDSG ist allerdings, dass kein Grund zur Annahme besteht, dass das schutzwürdige Interesse des Betroffenen am Ausschluss der Datenverwendung überwiegt. Hiervon ist jedoch beim Einsatz der oben genannten Beschäftigtendaten zur Qualitätssicherung nicht auszugehen, jedenfalls wenn folgende Bedingungen eingehalten werden:

- personenbezogene Verwendung nur intern oder durch Auftragsdatenverarbeiter,

- d.h. keine Übermittlung der personenbezogenen Beschäftigtendaten an (eigenständig verantwortliche) Dritte (darunter auch Studienportale und Datentreuhänder) und

- insbesondere keine Veröffentlichung;

- einrichtungsübergreifende Qualitätssicherung daher nur (nach außen) in anonymisierter Form (intern kann eine Zuordnungsmöglichkeit offen gehalten werden);

• Verwendung ausschließlich zur Qualitätssicherung, keine weiteren Zweckänderungen;

- insbesondere kein Einsatz zur individuellen Leistungs- oder Verhaltenskontrolle.

Das abstrakte Risiko der Verwendung entsprechender Daten im Arzthaftungs- bzw. allgemeiner einem Medizinschadensprozess oder bei einem entsprechenden Regress des Einrichtungsträgers gegen den Beschäftigten dürfte an der vorzunehmenden Interessenabwägung nichts ändern. ${ }^{935}$ Denn der Patientenbezug muss nach den Antworten auf die vorstehenden Fragen ohnehin möglichst frühzeitig beseitigt werden, so dass sich dieses Risiko kaum konkretisieren wird, denn ohne Patientenbezug besteht keine nachweisbare Beteiligung an einem Medizinschaden. Außerdem zählen die (generalisierenden) Qualitätssicherungsdaten nicht zur Patientenakte, in welche in aller Regel ein umfassendes Einsichtsrecht seitens des Patienten besteht ( $\$ 6309$ BCB). Datenschutzrechtliche Auskunftsansprüche ( $\$ 34$ BDSG) des Patienten würden dagegen greifen, solange noch Patientenbezug besteht, unterliegen aber eher Einschränkungen.

\section{Forschung}

Nach $\mathbb{2} 28$ Abs. 2 Nr. 3 BDSG ist die Übermittlung und Nutzung von Daten zu einem anderen als dem Erhebungszweck zulässig, wenn dies „im Interesse einer Forschungseinrichtung zur Durchführung wissenschaftlicher Forschung erforderlich ist, das wissenschaftliche Interesse an der Durchführung des Forschungsvorhabens das Interesse des Betroffenen an dem Ausschluss der Zweckänderung erheblich überwiegt und der Zweck der Forschung auf andere Weise nicht oder nur mit unverhältnismäßigem Aufwand erreicht werden kann“. Die hier genannten Voraussetzungen decken

934 Bei intendierten Beschäftigten-individuellen Rückmeldungen dürfte wie ausgeführt grundsätzlich $\$ 32$ BDSG greifen. 935 Vgl. insoweit aber die Diskussion um anonyme Meldesysteme für Fehler oder sonstige „critical incidents“. 
sich mit denen der bereits ausführlicher dargestellten Forschungsklausel für sensible Daten gemäß $\mathbb{2} 28$ Abs. 6 Nr. 4 BDSG ${ }^{936}$ mit zwei Ausnahmen:

- Zum einen werden vorliegend lediglich die Übermittlung und die Nutzung als erlaubte Datenverwendungsakte genannt,

- zum anderen muss dies „im Interesse einer Forschungseinrichtung“ erforderlich sein.

Daraus wird vielfach gefolgert, es sei nur sogenannte Drittforschung erlaubt. Wenn aber nun die erhebende Behandlungseinrichtung selbst eine Forschungseinrichtung ist, dann ist nach hier vertretener Auffassung auch die Eigenforschung auf Basis von $\mathbb{5} 28$ Abs. 2 Nr. 3 BDSG erlaubt, ${ }^{937}$ wobei Übermittlung und Nutzung insoweit wohl nur als Regelbeispiele auszulegen sind und - vor allem in Anbetracht der erlaubten, einschneidenden Übermittlung erst recht - auch andere Verwendungsakte zulässig sein können. Letztlich ist damit im Rahmen der genannten Vorschrift auch Verbundforschung erlaubt.

Die übrigen Tatbestandsvoraussetzungen unterscheiden sich vom gesetzlichen Ansatz her nicht von denen bei der Forschung mit Patientendaten und lassen sich wie folgt zusammenfassen:

- (Bestimmtes) Vorhaben der wissenschaftlichen Forschung

- Erforderlichkeit des Umgangs mit den gewünschten personenbezogenen Daten

- Angemessenheit des Datenumgangs (erhebliches Überwiegen des wissenschaftlichen Interesses)

- Praktische Alternativlosigkeit für die Erreichung des Forschungszwecks

Allerdings ist bei der Anwendung der entsprechenden Rechtsbegriffe zu berücksichtigen, dass Beschäftigtendaten üblicherweise eine geringere Sensibilität als Patientendaten aufweisen und daher im Ergebnis gerade im Rahmen der Abwägung (vor allem also bei der Angemessenheit, aber z.B. auch bei der praktischen Alternativlosigkeit) eher zu Gunsten der Forschung entschieden werden kann, gerade wenn es lediglich um die Eigenforschung der erhebenden wissenschaftlichen Forschungseinrichtung geht und zudem nicht die Beschäftigten ausgeforscht, sondern der Behandlungsprozess erforscht werden soll, es sich bei der Mitverarbeitung der Beschäftigtendaten also um eine Art „Kollateralschaden“ handelt und beiläufig über einen Beschäftigten gewonnene Erkenntnisse nicht oder jedenfalls nicht gegen diesen weiter verwendet werden. ${ }^{93^{8}}$ So wird man keineswegs immer, aber doch öfter als in Bezug auf Patientendaten auch von einem erheblichen Überwiegen des Forschungsinteresses ausgehen können.

Ansonsten könnte auch ein Wertungswiderspruch zu $₫ 28$ Abs. 2 Nr. 1 BDSG bestehen, der auf die sehr allgemeinen berechtigten Interessen der verantwortlichen Stelle nach $\mathbb{} 28$ Abs. 1 S. 1 Nr. 2 BDSG verweist, wo sogar ein einfaches Überwiegen genügt, obwohl wissenschaftliche Forschung vor verfassungsrechtlichem Hintergrund doch privilegiert sein sollte.

Auch vor diesem Hintergrund ist sogar zu erwägen, für die Eigenforschung der gleichzeitig behandelnden (und damit Behandlungsdaten erhebenden) und forschenden 
(die Behandlungsdaten hierfür weiter verwendenden) Einrichtung direkt auf $\mathbb{} 28$ Abs. 2 Nr. 1 BDSC zurückzugreifen, da dieser Erlaubnistatbestand insoweit als vorrangig gegenüber der vom Wortlaut her eher auf die Übermittlung an eine (externe) Forschungseinrichtung gemünzten Nr. 3 angesehen werden kann.

Im Ergebnis erscheint es vertretbar, jedenfalls für die reine Eigenforschung unter den schon zur Qualitätssicherung genannten Bedingungen, 939 eine gesetzliche Erlaubnis zum Umgang mit Beschäftigtendaten anzunehmen.

\section{Notwendigkeit und Möglichkeit einer individuellen Einwilligung}

Soweit nach den vorigen Ausführungen gesetzliche Erlaubnisnormen vorliegen, nach hier vertretener Auffassung also für einen restringierten internen Datenumgang, ist eine Einwilligung entbehrlich, so dass es nicht auf die teils umstrittene Frage ankommt, ob und inwieweit diese im Beschäftigungsverhältnis überhaupt wirksam erteilt werden kann. ${ }^{940}$ Zur weiteren Absicherung bei nicht ganz klarer Rechtslage könnte allerdings gleichwohl auf eine Einwilligung zurückgegriffen werden. Wenn die Einwilligung wirklich freiwillig und deren Verweigerung für den Beschäftigten nicht mit Nachteilen verbunden ist, sprechen nach hier vertretener Auffassung auch im Beschäftigungsverhältnis keine grundsätzlichen Bedenken gegen deren Zulässigkeit und Wirksamkeit.

Soweit keine gesetzliche Erlaubnisnorm vorliegt, was beispielsweise bei der personenbezogenen Übermittlung von Beschäftigtendaten für Zwecke der externen Qualitätssicherung oder der Drittforschung naheliegt, empfiehlt sich zur Absicherung eher eine kollektive Betriebs- bzw. Dienstvereinbarung denn eine individuelle Einwilligungslösung, wenn man sich nicht über die parallele Anwendung beider Handlungsinstrumentarien doppelt absichern möchte, da die kollektive Lösung wahrscheinlich praktikabler und überdies wohl auch rechtssicherer ist. ${ }^{941}$

Von einer datenschutzrechtlichen Einwilligung könnte man ohnehin nur bei einer zustimmenden Willensäußerung des Betroffenen selbst oder, soweit dieser nicht einwilligungsfähig ist, von dessen Vertreter sprechen. Von einer fehlenden Einwilligungsfähigkeit kann man beim behandelnden Personal allerdings kaum ausgehen, denn die Behandlung durch nicht-einwilligungsfähiges Personal muss ohnehin vermieden werden. Außerdem ist weder die Einrichtungsleitung noch der Betriebsrat ein Vertreter im eben genannten Sinn.

\subsubsection{Betriebs- bzw. Dienstvereinbarung als kollektive Alternative}

Eine Zustimmung von Einrichtungsleitung und Betriebsrat gilt damit nicht als Einwilligung für die Beschäftigten ${ }^{942}$, wohl aber, soweit dies in Form einer Betriebs- bzw. Dienstvereinbarung erfolgt, als Rechtsgrundlage eigener Art für den Umgang mit Beschäftigtendaten.

939 Zu den vergleichbaren Bedingungen bei der Qualitätssicherung oben S. $325 f$.

940 Vgl. zu dieser Streitfrage: Trittin/Fischer, NZA 2009, 343, 344, nach denen in erster Linie die Betriebsvereinbarung die gewünschte Rechtssicherheit bietet. Am rechtssichersten wäre freilich eine Kombination von kollektiver Betriebsvereinbarung und individueller Einwilligung.

941 S. sogleich Kap. I.15.2.1.3, S. $328 f$.

942 In diese Richtung ließe sich die Fragestellung missverstehen. 
Hintergrund hierfür ist, dass eine Betriebsvereinbarung (zwischen Betriebsrat und Unternehmensleitung) nach dem BetrVG - bzw. im öffentlichen Bereich (des Bundes) eine Dienstvereinbarung nach dem Personalvertretungsgesetz des Bundes (BPersVG)ebenfalls als datenschutzrechtliche Erlaubnis bezüglich des Umgangs mit Beschäftigtendaten (nicht Patientendaten) gilt. ${ }^{943}$ Eine solche Vereinbarung könnte hier zur Absicherung getroffen werden und hätte jedenfalls nach teils vertretener Auffassung eine höhere Rechtfertigungswirkung als die Einwilligung im Beschäftigungsverhältnis. 944

\subsubsection{Exkurs: Mitbestimmung bei möglicher Leistungs- oder Verhaltens- kontrolle}

Soweit der Umgang mit den Beschäftigtendaten zur Kontrolle von Leistung oder Verhalten der Beschäftigten genutzt werden kann, könnte eine Maßnahme vorliegen, die der Mitbestimmung des Betriebs- bzw. Personalrates unterliegt. Dies ist insbesondere denkbar, wenn die Ergebnisse der Qualitätssicherung noch auf den einzelnen Beschäftigten zu beziehen sind. Eine eventuelle Mitbestimmungspflicht ist unabhängig vom Vorliegen einer datenschutzrechtlichen Erlaubnis und damit keine Frage des Beschäftigtendatenschutzes, weshalb sie hier nur kurz als Exkurs dargestellt werden soll. Soweit eine Mitbestimmungspflicht vorliegt, müsste zusätzlich zur datenschutzrechtlichen Erlaubnis der Betriebsrat (bei privaten Stellen) bzw. der Personalrat (bei öffentlichen Stellen) oder die Mitarbeitervertretung (bei kirchlichen Stellen) zustimmen. Die Frage nach der Mitbestimmungspflicht soll vorliegend nur exemplarisch anhand des für Privatunternehmen, also auch Krankenhäuser in privater Trägerschaft, mit Betriebsrat einschlägigen Betriebsverfassungsgesetzes (BetrVG) untersucht werden.

Der Betriebsrat hat ein Mitbestimmungsrecht bei der Einführung und Anwendung von technischen Einrichtungen, die dazu bestimmt sind, das Verhalten oder die Leistung der Arbeitnehmer zu überwachen (\$ 87 Abs. 1 Nr. 6 BetrVG). Damit steht dem Betriebsrat in der Regel ein Mitbestimmungsrecht bei der Einführung von Personalinformationssystemen zu. Entscheidend für die Erforderlichkeit einer Mitbestimmung ist im Ergebnis aber nicht die Zielsetzung, sondern die objektive Eignung des technischen Systems (dazu zählen auch auf Computern laufende Programme) zur Mitarbeiterüberwachung. Eine technische Einrichtung, die aufgrund des verwendeten Programms Verhaltens- und Leistungsdaten auswertet, ist nach der Rechtsprechung des Bundesarbeitsgerichts zur Überwachung geeignet und dazu objektiv bestimmt. ${ }^{945}$ Unerheblich ist somit, ob das Verfahren der Datenverarbeitung auch tatsächlich zur Überwachung der Mitarbeiter eingesetzt wird, solange dies nur möglich und nicht ganz fernliegend ist.

Keine Voraussetzung für die Mitbestimmung ist es jedenfalls, dass der Arbeitgeber mit der Einführung oder Anwendung der technischen Einrichtung eine Überwachungsabsicht verfolgt. Das Gesetz verlangt lediglich, dass die Einrichtung zur Über-

943 Dix, in: Simitis (Hg.), BDSG, \& 1 Rdnr. 166; Scholz/Sokol, in: Simitis (Hg.), BDSG, \& 4 Rdnr. 11.

944 Zur Absicherung in erster Linie durch Betriebsvereinbarung: Trittin/Fischer, NZA 2009, 343, 344.

945 BAG, CR 1994, 111; Lembke in: Henssler/Willemsen/Kalb, Arbeitsrecht Kommentar, BDSG Einf., Rdnr. 81; kritisch Richardi in: Richardi (Hrsg.), BetrVG, 10. Aufl. 2006, §87 Rdnr. 501. 
wachung bestimmt ist, fordert also nicht zusätzlich, dass der Arbeitgeber die Aussagen über Verhalten oder Leistung einzelner Arbeitnehmer zu deren Überwachung verwendet. Keine Rolle spielt weiter, ob die Überwachung das Ziel der technischen Einrichtung oder nur ein Nebeneffekt ist und ob die Daten, die die technische Einrichtung liefert, zur Überwachung tatsächlich ausgewertet werden oder nicht. Es genügt, dass die Einrichtung Daten liefert, die (unmittelbar oder mittelbar) Rückschlüsse auf das Verhalten oder die Leistung der Arbeitnehmer ermöglichen. ${ }^{946}$ Damit unterliegt die automatisierte Verarbeitung von Beschäftigtendaten regelmäßig der Mitbestimmungspflicht des Betriebsrates nach $₫ 87$ Abs. 1 Nr. 6 BetrVG.

Folglich unterliegt auch eine Sekundärnutzung von Behandlungsdaten, wenn deren Ergebnisse (zunächst gleich, ob im Rahmen von Forschungsvorhaben oder Qualitätssicherungsmaßnahmen) Rückschlüsse auf die Qualität der durch einen Beschäftigten erfolgten Behandlung (und damit dessen Leistung) zulassen, der Mitbestimmung des Betriebsrates.

Bei der Forschung könnte man dem entgegenhalten, dass diese der Fortentwicklung des Standes der (medizinischen) Wissenschaft dienen und es einem Beschäftigten nicht als Schlechtleistung oder gar unangebrachtes Verhalten angelastet werden kann, wenn er „nur“ nach dem aktuellen Stand der medizinischen Wissenschaft behandelt. Zudem ist die Forschung über Art. 5 Abs. 3 GG grundrechtlich besonders geschützt. Daher könnte es nach summarischer Prüfung vertretbar sein, die Forschung, auch soweit sie einen - allerdings nur indirekten, nicht intendierten und nicht weiterverwerteten - Bezug zu Leistung oder Verhalten von (bestimmbaren) Beschäftigten hat, aus der Mitbestimmungspflicht auszunehmen.

Für die beschäftigtenbezogene Qualitätssicherung, also bei jedenfalls nicht fern liegender Möglichkeit der Feststellung, ob ein Beschäftigter nach den gültigen Qualitätsstandards behandelt hat, wird man aber wohl eine Mitbestimmungspflicht annehmen müssen.

Soweit der Betriebsrat also einerseits ohnehin mitzubestimmen hat und andererseits eine Betriebsvereinbarung eine datenschutzrechtliche Erlaubnisnorm darstellen kann, kann es sich empfehlen, offensiv den Abschluss einer solchen Vereinbarung anzustreben. Am rechtssichersten wäre freilich eine Kombination von kollektiver Betriebsvereinbarung und individueller Einwilligung, wobei zumindest letzteres nicht zwingend erforderlich erscheint.

Dieser Exkurs wird auf der Ebene der Personalvertretungsgesetze des Bundes und der Länder sowie der Mitarbeitervertretungsgesetze der Kirchen nicht fortgesetzt. Es ist jedoch nicht unwahrscheinlich, dass auch diese Gesetze, die grundsätzlich auch für die den entsprechenden Trägern zugeordneten Kliniken gelten, vergleichbare Regelungen treffen.

\subsection{Anwendungsbereich der Landesdatenschutzgesetze}

Die Rechtslage nach den Landesdatenschutzgesetzen (LDSG) stellt sich nach summarischer Prüfung dem BDSG im Ergebnis vergleichbar dar.

946 Lembke in: Henssler/Willemsen/Kalb, Arbeitsrecht Kommentar, 3. Aufl. 2008, BDSG Einf., Rdnr. 81; eher die Ermöglichung unmittelbarer Rückschlüsse fordernd: Richardi in: Richardi (Hrsg.), BetrVG, $\$ 87$ Rdnr. $504 f$. 
Alle Bundesländer bis auf Bayern haben in ihre LDSG für die Beschäftigten im öffentlichen Dienst inzwischen auch einen speziellen Paragrafen zur Datenverarbeitung in Dienst- und Arbeitsverhältnissen eingefügt. Diese enthalten neben der Erlaubnis zum Datenumgang für die Begründung, Durchführung und Beendigung von Beschäftigungsverhältnissen entweder Öffnungsklauseln für andere Aufgaben ${ }^{947}$ oder sind wie $\mathbb{3 2}$ BDSG nicht abschließend, so dass auf andere Erlaubnistatbestände wie die Notwendigkeit zur Erfüllung anderer Aufgaben, die Wahrung berechtigter Interessen sowie die Durchführung von Forschungsvorhaben zurückgegriffen werden kann. ${ }^{948}$ Teils wird sogar die Einwilligung explizit als Erlaubnis zugelassen. ${ }^{949}$

In Bayern ist für Beschäftigungsverhältnisse im öffentlichen Bereich mangels spezieller Norm ohnehin auf allgemeine Erlaubnistatbestände zurückzugreifen.

Auch Dienstvereinbarungen nach den Landespersonalvertretungsgesetzen gelten insoweit als Rechtsvorschriften für den Umgang mit Beschäftigtendaten oder werden explizit als Erlaubnis eigener Art ${ }^{950}$ genannt. Soweit die einschlägigen Erlaubnistatbestände für den geplanten Datenumgang nicht ausreichen, insbesondere weil beschäftigtenbezogene Übermittlungen stattfinden sollen, bietet sich der Abschluss einer Dienstvereinbarung an, welche allgemein zur Absicherung zu empfehlen ist.

\subsection{Anwendungsbereich der kirchlichen Datenschutzgesetze}

\subsubsection{Kliniken der evangelischen Kirche}

Im Bereich der evangelischen Kirche (Evangelische Kirche in Deutschland - EKD) ent-

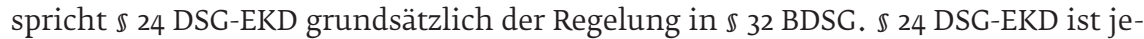
doch viel detaillierter und umfangreicher als $\$ 32$ BDSG. So werden in $\mathbb{2} 24 \mathrm{Abs}$. 1 DSGEKD ausdrücklich die Durchführung organisatorischer, personeller und sozialer Maßnahmen, insbesondere auch zu Zwecken der Personalplanung und des Personaleinsatzes, genannt. Als solche personelle Maßnahmen sind dabei auch Maßnahmen der Qualitätssicherung mit Beschäftigtenbezug anzusehen. Außerdem werden in $\mathbb{2} 24$ Abs. 1 DSG-EKD ausdrücklich Tarifverträge und Dienstvereinbarungen als Erlaubnistatbestände genannt.

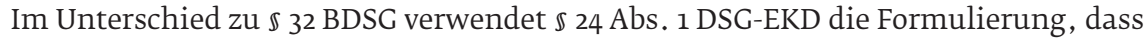
kirchliche Stellen mit Daten ihrer Beschäftigen „nur“ zu den genannten Zwecken umgehen dürfen, was den Eindruck erweckt, die Norm habe im Gegensatz zu $\mathbb{3} 32$ BDSG Ausschließlichkeitscharakter. Da jedoch im Gegensatz zu $₫ 32$ BDSC die Datenverarbeitung ausdrücklich erlaubt ist, wenn „eine Rechtsvorschrift“ diese vorsieht, besteht im Ergebnis kein Unterschied, d.h. neben $\$ 24$ DSG-EKD können auch andere Erlaubnistatbestände herangezogen werden. Hier kommt für Forschungszwecke vor allem $₫ 5$ Abs. 2 Nr. 9 DSG-EKD in Betracht.

947 Vgl. z.B. $\$ 36$ Abs. 1 LDSG BW: „Durchführung innerdienstlicher planerischer, organisatorischer, personeller, sozialer oder haushalts- und kostenrechnerischer Maßnahmen“. Dieser Passus findet sich in den LDSG der meisten Bundesländer und dürfte Maßnahmen der internen Qualitätssicherung umfassen.

948 Teils wird ergänzend explizit auf die Landesbeamtengesetze verwiesen, so in $\S 20$ Abs. 1 LDSG HB.

949 So $\$ 29$ Abs. 1 S. 1 LDSG BB für die Datenübermittlung an Stellen außerhalb des öffentlichen Bereichs, woraus a majore ad minus auf eine grundsätzlich Zulässigkeit der Einwilligung in interne Datenverarbeitung geschlossen werden kann.

So wiederum Z.B. \$36 Abs. 1 LDSG BW. 
$\mathbb{} 24$ Abs. 2 DSG-EKD regelt die Übermittlung von Beschäftigtendaten an Stellen außerhalb des kirchlichen Bereichs, wobei auch hier die Einschränkung „nur“ vorgenommen wird, so dass damit nicht auf andere Erlaubnistatbestände zurückgegriffen werden kann. Bei Forschungszwecken kommt vor allem $\$ 24$ Abs. 2 Nr. 1 DSG-EKD in Betracht, der die Darlegung eines überwiegenden rechtlichen Interesses durch die empfangende Stelle erfordert. Hier erscheint jedoch besonders zweifelhaft, ob ein Forschungsinteresse als ein „rechtliches“ angesehen werden kann. Auch kommt $\$ 24$ Abs. 2 Nr. 2 DSG-EKD in Betracht, der die Übermittlung zulässt, wenn Art oder Zielsetzung der dem Beschäftigten übertragenen Aufgaben dies erfordert. Dies kann gegeben sein, wenn Mitarbeiter von vornherein im Forschungsbereich eingesetzt werden. $\mathbb{S} 24$ Abs. 2 Nr. 3 DSG-EKD kommt vorliegend nicht in Betracht, weil weder die Forschung noch die Qualitätssicherung im Interesse des Beschäftigten, der mit der „betroffenen Person“ gemeint ist, liegen, dies aber jedenfalls nicht „offensichtlich“ ist.

\subsubsection{Kliniken der katholischen Kirche}

In Kliniken katholischer Träger ist für den Beschäftigtendatenschutz zunächst $\mathbb{1} 10 \mathrm{a}$ KDO ${ }^{951}$ einschlägig. Dieser entspricht weitgehend wörtlich $\mathbb{3} 32$ BDSC mit der Ausnahme, dass von den Daten eines Beschäftigten explizit „Daten über die Religionszugehörigkeit, die religiöse Überzeugung und die Erfüllung von Loyalitätsobliegenheiten" mit umfasst sind, was sich auf den vorliegenden Kontext aber nicht auswirkt. $\mathbb{} 2 \mathrm{Abs} .12 \mathrm{KDO}$ definiert lediglich den Begriff des Beschäftigten. $\mathbb{s}$ 1oa Abs. $1 \mathrm{KDO}$ stellt wie $\mathbb{3} 32$ BDSG auf die Zweckbestimmung des Beschäftigungsverhältnisses ab, lässt aber den Rückgriff auf andere Erlaubnistatbestände der KDO unberührt.

Der Rückgriff auf diese ist somit immer dann zulässig und möglich, wenn $\mathbb{1} 10 a$ KDO als Erlaubnistatbestand für die Weitergabe von Beschäftigtendaten nicht ausreichend ist. Dies ist in den gleichen Fällen gegeben, in denen auch $\mathbb{3} 32$ BDSG keine ausreichende Grundlage darstellt, somit insbesondere bei Forschungszwecken und bei Maßnahmen der Qualitätssicherung, die eine beschäftigtenbezogene Leistungskontrolle ermöglichen, aber nicht beabsichtigen.

Dabei entspricht $\$ 28$ Abs. 1 BDSG, der die Datenverarbeitung zu eigenen Zwecken regelt, $\mathbb{1} 10 \mathrm{Abs}$. $1 \mathrm{KDO}$. Danach ist das Speichern, Verändern oder Nutzen personenbezogener Daten zulässig, wenn es zur Erfüllung der in der Zuständigkeit der verantwortlichen Stelle liegenden Aufgaben erforderlich ist und es für die Zwecke erfolgt, für die die Daten erhoben worden sind. Eine $\mathbb{} 28$ Abs. 1 Satz 1 Nr. 2 BDSG vergleichbare Norm, die auf die Wahrnehmung berechtigter Interessen der verantwortlichen Stelle und eine Interessenabwägung abstellt, besteht dabei in der KDO nicht ausdrücklich. Qualitätssichernde Maßnahmen können jedoch als Aufgaben der verantwortlichen Stellen nach $\mathbb{1} 10$ Abs. 1 KDO angesehen werden. Eine $\mathbb{2} 28$ Abs. 1 Satz 1 Nr. 2 BDSG entsprechende Interessenabwägung kann jedoch in der KDO nicht verortet werden. Insbesondere können qualitätssichernde Maßnahmen nicht als Orga-

951 Hier wird das Muster des neuen $§ 10 a$ KDO zugrunde gelegt, wie inn die Diözesanvollversammlung mit Beschluss vom 18.11.2013 beschlossen hat und wie er beispielsweise schon von den Bistümern Hamburg und Fulda umgesetzt wurde. Mit einer Umsetzung durch alle Bistümer ist zeitnah zu rechnen. 
nisationsuntersuchungen nach $₫$ 10 Abs. 3 KDO angesehen werden. Diese Norm besagt jedoch nur, dass solche Untersuchungen keine Zweckänderung darstellen.

Dem die Datennutzung zu Forschungszwecken regelnden $\mathbb{2} 28$ Abs. 2 Nr. 3 BDSG entspricht $\mathbb{1} 10$ Abs. 2 Nr. 9 KDO, der bis auf die Formel „im Interesse einer Forschungseinrichtung“"wortgleich ist. Dabei regelt $\$ 10 \mathrm{Abs} .2$ KDO die Datennutzung bei Zweckänderung, während $\$ 10$ Abs. 1 KDO von einer Nutzung im Rahmen der Zweckerhebung ausgeht. Die Sekundärnutzung von Beschäftigtendaten in Patientenakten zu Forschungszwecken ist somit auch unter der KDO unter Einhaltung einer Interessenabwägung grundsätzlich zulässig.

Während $\$ 28$ BDSG neben der Datenerhebung und -speicherung sogleich auch deren Übermittlung regelt, wird diese in $\mathbb{s} 10 \mathrm{KDO}$ nicht genannt. Vielmehr wird diese in den $\mathbb{\$} 11$, 12 KDO geregelt, wobei $\mathbb{1} 11$ KDO die Weitergabe an kirchliche und öffentliche Stellen und $\$ 12 \mathrm{KDO}$ an nicht-kirchliche und nicht-öffentliche Stellen regelt. Dabei verweisen beide Normen zunächst auf die Voraussetzungen des $\mathbb{1} 10$ KDO und stellen weiter darauf ab, dass die Datenübermittlung zur Aufgabenerfüllung der übermittelnden Stelle (bei $\mathbb{1 1}$ KDO alternativ der empfangenden Stelle) erforderlich ist. $\mathbb{S} 12$ Abs. 1 Nr. 2 KDO kommt dabei vorliegend keine Bedeutung zu. Denn dort wird auf ein Interesse der empfangenden Stelle abgestellt. Die Qualitätssicherung liegt jedoch immer nur im Interesse der übermittelnden Stelle. Bei der Forschung wäre zwar ein Interesse des Empfängers zu bejahen, sie wird aber schon über den Verweis auf $\mathbb{S} 10$ in $\mathbb{1} 12 \mathrm{Abs}$. 1 Nr. 1 KDO erfasst. Beides, Qualitätssicherung und Forschung, wird daher über den Verweis auf $\mathbb{1} 10$ erfasst. Ein Rückgriff auf $\mathbb{1} 12 \mathrm{Abs}$. 1 Nr. 2 KDO wäre daher nur dann erforderlich, wenn die Voraussetzungen des $\mathbb{1}$ 10 KDO nicht vorliegen.

Nach $\$$ 10a Abs. 3 KDO bleiben die Beteiligungsrechte nach der jeweils geltenden Mitarbeitervertretungsordnung (der Interessenvertretungen der Beschäftigten der katholischen Kirche) unberührt, so dass auch hier entsprechende Dienstvereinbarungen zur Absicherung herangezogen werden können. 